Bundesgesundheitsbl 2020 - 63:228-241 https://doi.org/10.1007/s00103-019-03077-8 (c) Springer-Verlag GmbH Deutschland, ein Teil von Springer Nature 2020

\section{Inhaltsverzeichnis}

1. Einleitung, Definitionen und Hintergrund

1.1. Zielgruppe dieser Empfehlung

1.2. Bezug zu vorausgegangenen Empfehlungen der KRINKO

2. Ziel der Surveillance

3. Evidenz für die Surveillance als Methode zur Reduktion von nosokomialen Infektionen

4. Surveillance-Methoden für das interne Qualitätsmanagement

4.1. Externe versus eigene SurveillanceMethoden und -Falldefinitionen

4.2. Definitionen für nosokomiale Infektionen für die Surveillance

4.3. Surveillance-Personal

4.4. Erkennung von Infektionsfällen (Zählerdaten) mit Hilfe von Indikatoren

4.5. Bestimmung der Bezugsdaten (Nennerdaten)

4.6. Surveillance-Protokolle

4.7. Orientierung an nationalen Daten

4.8. Unterstützung durch die Informatik und das Controlling

5. Auswahl der Infektionsarten und Bereiche für die Surveillance in der eigenen Einrichtung

5.1. Auszuwählende Bereiche

5.2. Auszuwählende Infektionen

6. Interpretation von Surveillance-Daten

7. Hinweise zum Feedback von SurveillanceDaten

8. Evaluation der Surveillance in der eigenen Einrichtung

9. Surveillance im Sinne des internen Qualitätsmanagements versus externe Qualitätssicherung

10. Empfehlungen

Literatur

Anhang: Glossar

\title{
Surveillance von nosokomialen Infektionen
}

\author{
Empfehlung der Kommission \\ für Krankenhaushygiene und \\ Infektionsprävention (KRINKO) beim \\ Robert Koch-Institut
}

\section{Einleitung, Definitionen und Hintergrund}

Unter "Surveillance“ von nosokomialen Infektionen ist die fortlaufende, systematische Erfassung, Analyse und Interpretation der Daten zu diesen Infektionen, die zur Planung, Einführung und Evaluation von medizinischen Maßnahmen notwendig sind, zu verstehen. Dazu gehört die aktuelle Übermittlung der Daten an diejenigen, die diese Informationen benötigen [1].

Die Pflicht zur Surveillance wurde in Deutschland im Infektionsschutzgesetz (IfSG) festgelegt: gemäß $\$ 23$ Abs. 4 der Novellierung des IfSG aus dem Jahr 2011 haben die Leiter ${ }^{1}$ von Krankenhäusern, Einrichtungen für ambulantes Operieren und Vorsorge- und Rehabilitationseinrichtungen, in denen eine den Krankenhäusern vergleichbare medizinische Versorgung erfolgt, sicherzustellen, dass die nach Absatz 4a festgelegten nosokomialen Infektionen fortlaufend in einer gesonderten Niederschrift aufgezeichnet, bewertet und sachgerechte Schlussfolgerungen hinsichtlich erforderlicher Präventionsmaßnahmen gezogen werden und die erforderlichen Präventionsmaßnahmen dem Personal mitgeteilt und umgesetzt werden. Nach Absatz 4a hat das Robert KochInstitut (RKI) entsprechend den jeweiligen epidemiologischen Erkenntnissen die nach Absatz 4 zu erfassenden nosokomialen Infektionen festzulegen. Die Festlegungen hat es in einer Liste im Bundes-

\footnotetext{
Grundsätzlich sind in diesem Dokument bei allen Berufs- bzw. Gruppenbezeichnungen immer alle Geschlechter gemeint.
}

gesundheitsblatt zu veröffentlichen. Das erfolgte zuletzt im Jahr 2013 [2].

Nachfolgend gibt die Kommission für Krankenhaushygiene und Infektionsprävention (KRINKO) Empfehlungen zur praktischen Umsetzung des $\$ 23$ Abs. 4 IfSG und ergänzt damit die o.g. Veröffentlichung des RKI. Im Sinne ihrer Zuständigkeit beschränkt sich die KRINKO hier auf die Empfehlungen zur Umsetzung bei der Surveillance von nosokomialen Infektionen; Empfehlungen zur Surveillance von Krankheitserregern mit speziellen Resistenzen und Multiresistenzen sind nicht Gegenstand dieser Empfehlung und von Seiten der KRINKO auch nicht geplant.

Diese Empfehlung bezieht sich nur auf die Anwendung der Surveillance für das interne Qualitätsmanagement. Darüber hinausgehende Verpflichtungen zur Dokumentation nosokomialer Infektionen, wie zum Beispiel die Dokumentation postoperativer Wundinfektionen nach Vorgabe der externen Qualitätssicherung (Institut für Qualitätssicherung und Transparenz im Gesundheitswesen, IQTIG) unterliegen anderen Prinzipien und sind nicht Gegenstand dieser Empfehlung (s. Kapitel 9).

Aktuell ist Surveillance in Deutschland in erster Linie in Krankenhäusern standardisiert etabliert, da mit dem Krankenhaus-Infektions-Surveillance-System (KISS) stratifizierte nationale Referenzdaten für diesen Bereich vorliegen. Es existiert ein Surveillance-Protokoll für postoperative Wundinfektionen im ambulanten Bereich [3]. Wegen der erheblichen Variabilität der Patientenpopulationen in ambulanten Einrichtungen steht hier vor allem die Surveillance im longi- 
Tab. 1 Kategorien in der Richtlinie für Krankenhaushygiene und Infektionsprävention (2010) Kategorie Bewertungsgrundlage

Kategorie IA Diese Empfehlung basiert auf gut konzipierten systematischen Reviews oder einzelnen hochwertigen randomisierten kontrollierten Studien.

Kategorie IB Diese Empfehlung basiert auf klinischen oder hochwertigen epidemiologischen Studien und strengen, plausiblen und nachvollziehbaren theoretischen Ableitungen.

Kategorie II Diese Empfehlung basiert auf hinweisenden Studien/Untersuchungen und strengen, plausiblen und nachvollziehbaren theoretischen Ableitungen.

Kategorie III Maßnahmen, über deren Wirksamkeit nur unzureichende oder widersprüchliche Hinweise vorliegen, deshalb ist eine Empfehlung nicht möglich.

Kategorie IV Anforderungen, Maßnahmen und Verfahrensweisen, die durch allgemein geltende Rechtsvorschriften zu beachten sind.

tudinalen Ansatz im Vordergrund, d.h. dass man die Infektionsraten der eigenen Einrichtung über die Zeit beobachtet und analysiert.

\subsection{Zielgruppe dieser Empfehlung}

Diese Empfehlung richtet sich primär an die Leiter von Krankenhäusern, Einrichtungen für ambulantes Operieren sowie Vorsorge- und Rehabilitationseinrichtungen mit vergleichbarer medizinischer Versorgung, sowie an das Hygienefachpersonal und weitere interessierte Mitarbeiter dieser Einrichtungen. Darüber hinaus sollte auch für ausgewählte weitere Bereiche der ambulanten Medizin, z.B. Dialyse- oder Heimbeatmungseinrichtungen, ein Surveillance-System etabliert werden.

Ambulante Einrichtungen können in dieser Empfehlung nicht in jeder Hinsicht adressiert werden, sie können die vorliegende Empfehlung aber nutzen, um eine an die Einrichtung angepasste Surveillance zu entwickeln.

\subsection{Bezug zu vorausgegangenen Empfehlungen der KRINKO}

Diese Empfehlung ersetzt die KRINKOEmpfehlung zur Surveillance von nosokomialen Infektionen (Umsetzung von $\$ 23$ IfSG) aus dem Jahr 2001 und die Empfehlung zur Surveillance von postoperativen Wundinfektionen in Einrichtungen für das ambulante Operieren $[4,5]$. Surveillance gehört zu den acht von der Weltgesundheitsorganisation (WHO) definierten Kernkomponenten der Infektionsprävention im Krankenhaus [6]. Des- halb hat diese Empfehlung Bezug zu allen KRINKO-Empfehlungen, in denen es um die Prävention von spezifischen nosokomialen Infektionen geht.

Die hier aufgeführten Empfehlungen sind zum Teil mit Evidenzkategorien entsprechend der Mitteilung „Die Kategorien in der Richtlinie für Krankenhaushygiene und Infektionsprävention - Aktualisierung der Definitionen“ von 2010 versehen (• Tab. 1 ; [7]).

\section{Ziel der Surveillance}

Ziel der Surveillance ist die Reduktion nosokomialer Infektionen in den genannten Gesundheitseinrichtungen. Deshalb ist entscheidend, dass die Surveillance-Daten regelmäßig und anlassbezogen sowie in hoher Qualität an das medizinische Personal der Stationen und Abteilungen zurückgespiegelt werden, für die die Surveillance durchgeführt wird. Nationale Referenzdaten haben in diesem Zusammenhang einen hohen Stellenwert, da hierdurch ein Vergleich mit ähnlichen Stationen/Abteilungen unter vergleichbaren Rahmenbedingungen möglich ist. Das Feedback für die Stationen und Abteilungen trägt auch durch den Vergleich mit nationalen Referenzdaten zur Motivation bei.

Ca. $95 \%$ aller nosokomialen Infektionen treten als „endemische Infektionen“ (siehe Glossar) auf $[8,9]$. Das Personal der einzelnen Stationen und Abteilungen hat sich häufig an dieses endemische Niveau von nosokomialen Infektionen gewöhnt, deshalb werden sie oft nicht als Problem wahrgenommen. Surveillance zielt in ers- ter Linie auf die Reduktion der endemischen nosokomialen Infektionen ab.

Die Identifikation von Ausbrüchen nosokomialer Infektionen ist hingegen nicht das primäre Ziel der Surveillance, da diese in der Regel durch andere Ansätze schneller erkannt werden. Zum Vorgehen bei Ausbrüchen wird auf die Empfehlung der KRINKO „Ausbruchmanagement und strukturiertes Vorgehen bei gehäuftem Auftreten nosokomialer Infektionen“ verwiesen [10].

In diesem Kontext muss beachtet werden, dass es auch bei Umsetzung aller bekannter Präventionsmaßnahmen nicht möglich ist, alle nosokomialen Infektionen zu vermeiden.

Surveillance ist integraler Bestandteil des internen Qualitätsmanagements nach dem „Plan-Do-Check-Act“-Zyklus, der den kontinuierlichen, mehrstufigen Ansatz im Qualitätsmanagement verdeutlicht [11]. Wenn ein Problem identifiziert wird, soll eine lösungsorientierte Problemanalyse erfolgen. Auf dieser Basis müssen geeignete Interventionen gefunden werden, um die Lösungen $\mathrm{zu}$ implementieren. Surveillance-Daten liefern den Ausgangspunkt für diesen Prozess und können im Anschluss den Erfolg von eingeleiteten Interventionsmaßnahmen belegen.

Darüber hinaus führen SurveillanceMaßnahmen zur Quantifizierung nosokomialer Infektionen und damit zu einer deutlichen Aufmerksamkeitssteigerung für dieses Problem. Dementsprechend hat die Surveillance folgende wesentliche Ziele:

- Steigerung der Aufmerksamkeit für das Problem der nosokomialen Infektionen

- Gezielte Identifikation von Problemen als Basis für deren Analyse und die Intervention

- Evaluation von eingeführten Interventionen

\section{Evidenz für die Surveillance als Methode zur Reduktion von nosokomialen Infektionen}

Surveillance soll zur Senkung von nosokomialen Infektionsraten führen und damit einen präventiven Effekt haben. Dabei kann die Reduktion nosokomialer Infek- 
tionen durch den sogenannten Hawthorne-Effekt zustande kommen (unter dem Bewusstsein der Beobachtung verbessert sich häufig die Arbeitsleistung), vor allem aber durch die gezielte Identifikation von Infektionsproblemen und anschließender Analyse und Intervention. In jedem Fall ist sie ein effektiver Motor für Anstrengungen zur Prävention von nosokomialen Infektionen.

Der Nachweis der Effektivität von Surveillance zur Infektionsprävention wurde im Rahmen einer großen kontrollierten Studie (SENIC, Study on the Efficacy of Nosocomial Infection Control) in den siebziger Jahren in den USA erbracht. In der Studie wurde festgestellt, dass Krankenhäuser mit intensiven Anstrengungen auf dem Gebiet der Surveillance und Infektionskontrolle geringere Infektionsraten aufwiesen [12]. Inzwischen konnte in Kohortenstudien aus vielen Ländern (überwiegend basierend auf Daten der nationalen Surveillance-Systeme) gezeigt werden, dass die Surveillance von nosokomialen Infektionen zu deren signifikanter Reduktion führt. Das betrifft die häufigen Infektionen wie postoperative Wundinfektionen [13-19], primäre Blutstrominfektionen (BSI) [20-23], beatmungsassoziierte Pneumonien [23, 24], katheterassoziierte Harnwegsinfektionen [25] und Clostridioides difficile-Infektionen (CDI) [26, 27].

In einem systematischen Review wurden Kohortenstudien, die den Einfluss der Surveillance auf das Auftreten von nosokomialen Infektionen untersucht haben, zusammenfassend analysiert [28]. Fünfundzwanzig Studien wurden in dieses Review eingeschlossen. Kontinuierliche Surveillance führte zu einer Reduktion der Infektionsraten um 5 bis $57 \%$. Kohortenstudien haben den Nachteil, dass sich im Verlauf der Studie neben der Einführung der Surveillance auch andere Einflussfaktoren verändern können. In zwei Studien konnte gezeigt werden, dass der Effekt der Surveillance unabhängig von den untersuchten Zeiträumen war [29, 30]. Verschiedene systematische Literaturanalysen haben sich mit der wissenschaftlichen Evidenz für einen genuinen, infektionspräventiven Effekt der Surveillance von nosokomialen Infektionen befasst; sie er- lauben zusammengefasst nach der KRINKO-Systematik die Zuordnung zur Kategorie IB [31, 32]. Kontrollierte Studien zum Effekt der Surveillance wurden seit der SENIC-Studie nicht publiziert.

Prospektive randomisierte kontrollierte Studien zur Effektivität bzw. Überlegenheit bestimmter Surveillance-Methoden liegen aktuell nicht vor. Deshalb haben alle folgenden Empfehlungen zu bestimmten Einzelmaßnahmen im Rahmen der Surveillance einen „Best Practice-Charakter“, oder die Evidenz kann nur aus einzelnen Kohortenstudien abgeleitet werden.

\section{Surveillance- Methoden für das interne Qualitätsmanagement}

\subsection{Externe versus eigene Surveillance-Methoden und -Falldefinitionen}

Die Surveillance-Methoden müssen sich an der Zielsetzung orientieren. Dabei kann es „die“ ideale Surveillance-Methode nicht geben, da im Hinblick auf die spezielle Patientenpopulation, die vorhandenen Probleme und die Aufwand-Nutzen-Relation die jeweils sinnvollste Methode angewendet werden sollte.

Vor allem in Risikobereichen ist es sinnvoll, sich etablierten SurveillanceSystemen wie dem Krankenhaus-Infektions-Surveillance-System (KISS) anzuschließen, um sich an deren Daten orientieren zu können. Teilweise verwenden die Einrichtungen die Methoden und Definitionen von KISS, ohne selbst am Surveillance-System teilzunehmen.

Unter Umständen kann es aber auch sinnvoll sein, eine eigene SurveillanceMethode zu etablieren, um der speziellen eigenen Patientenpopulation bzw. den existierenden spezifischen Problemen besser gerecht zu werden. Darüber hinaus existieren nicht für alle Infektionsarten und Problembereiche geeignete Surveillance-Protokolle im Rahmen von KISS. Ambulante Einrichtungen können beispielsweise nicht in jedem Bereich auf stratifizierte Referenzdaten zurückgreifen. Die Entwicklung einer eigenen Surveillance-Methode erfordert aber entsprechende Erfahrung und Sachverstand $[33,34]$. - Tab. 2 zeigt die Vorund Nachteile der drei Herangehensweisen.

Grundsätzlich sind Surveillance-Projekte mit ausreichendem zeitlichen Vorlauf in der jeweiligen Abteilung anzukündigen und gemeinsam vorzubereiten. Die Methoden sollen vorab gegenüber dem Behandlungsteam auch im Hinblick auf zu erwartende Limitationen transparent gemacht werden. Dabei sollte hervorgehoben werden, dass das Hygienefachpersonal und das Behandlungsteam gemeinsame Ziele im Hinblick auf die Infektionsprävention und den Patientenschutz verfolgen.

\subsection{Definition von nosokomialen Infektionen für die Surveillance}

Die für die praktische Surveillance anzuwendenden Definitionen für nosokomiale Infektionen unterscheiden sich von der im IfSG $\$ 2$ genannten Definition (nosokomiale Infektion = eine Infektion mit lokalen oder systemischen Infektionszeichen als Reaktion auf das Vorhandensein von Erregern oder ihrer Toxine, die im zeitlichen Zusammenhang mit einer stationären oder einer ambulanten medizinischen Maßnahme steht, soweit die Infektion nicht bereits vorher bestand). Die Definitionen für Surveillance-Zwecke zielen darauf $a b$, eine Vergleichbarkeit der Surveillance-Daten auf nationaler Ebene herzustellen. Es handelt sich hierbei um rein epidemiologische Definitionen zur Festlegung von nosokomialen Infektionen [35].

Weltweit haben sich seit langem für den Zweck der Surveillance die Definitionen der Centers for Disease Control and Prevention (CDC) etabliert, die erstmalig 1988 publiziert wurden [36]. Die Definitionen des European Centre for Disease Prevention and Control (ECDC) für nosokomiale Infektionen basieren weitgehend auf den CDC-Definitionen, deshalb sind die Infektionsraten weitestgehend miteinander vergleichbar (s. Infobox [37]).

Infektionen, bei denen die ersten Infektionszeichen bereits vor der Aufnahme in das Krankenhaus oder an Tag 1 oder Tag 2 des Krankenhausaufenthaltes vorhanden sind, werden im Sinne der Surveillance 


\begin{tabular}{|c|c|c|}
\hline Herangehensweise & Vorteile & Nachteile \\
\hline Teilnahme an KISS-Modulen & $\begin{array}{l}\text { - Protokolle inklusive Definitionen sind verfügbar und } \\
\text { anerkannt } \\
\text { - Etabliertes Schulungskonzept und Schulungspersonal } \\
\text { vorhanden, um die Anwendung der Definitionen regel- } \\
\text { mäßig zu trainieren } \\
\text { - Dateneingabesysteme und Systeme zur Berechnung der } \\
\text { eigenen Infektionsraten sind webbasiert verfügbar } \\
\text { - Referenzdaten sind verfügbar/ein Vergleich mit anderen } \\
\text { Einrichtungen ist möglich } \\
\text { - Qualitätssicherungskriterien sind vorhanden }\end{array}$ & $\begin{array}{l}\text { Protokolle sind nicht für alle Patientengruppen und } \\
\text { Infektionsarten verfügbar } \\
\text { - Teilweise sind die eigenen Patienten nicht ver- } \\
\text { gleichbar mit denen der übrigen teilnehmenden } \\
\text { Einrichtungen }\end{array}$ \\
\hline $\begin{array}{l}\text { Nutzung der KISS-Methoden } \\
\text { und Definitionen ohne } \\
\text { Teilnahme an KISS }\end{array}$ & $\begin{array}{l}\text { - Protokolle inklusive Definitionen sind verfügbar und } \\
\text { anerkannt } \\
\text { - Keine Teilnahme an Einführungskursen oder Erfahrungs- } \\
\text { austauschen erforderlich }\end{array}$ & $\begin{array}{l}\text { Kein regelmäßiges Training zum Umgang mit den } \\
\text { Definitionen (Validität des Vergleichs an Referenz- } \\
\text { daten?) } \\
\text { - Kein Dateneingabesystem und keine webbasierte } \\
\text { Berechnung der Infektionsraten }\end{array}$ \\
\hline $\begin{array}{l}\text { Verwendung selbst entwi- } \\
\text { ckelter Protokolle/Definiti- } \\
\text { onen }\end{array}$ & $\begin{array}{l}\text { Ggf. höhere Akzeptanz der Surveillance-Ergebnisse, } \\
\text { wenn eigene Definitionen bzw. Protokolle verwendet } \\
\text { werden } \\
\text { - Möglichkeit der Berücksichtigung besonderer Risikofak- } \\
\text { toren der eigenen Patientengruppe }\end{array}$ & $\begin{array}{l}\text { Keine Referenzdaten vorhanden, es sind nur Verglei- } \\
\text { che über die Zeit oder eventuell mit Daten aus der } \\
\text { Literatur möglich } \\
\text { - Sehr aufwendig und fehleranfällig, da für die Ent- } \\
\text { wicklung von Surveillance-Modulen viel Erfahrung } \\
\text { notwendig ist }\end{array}$ \\
\hline
\end{tabular}

\section{Infobox}

Allgemeine Definition einer nosokomialen Infektion nach KISS, National Healthcare Safety Network (NHSN) und ECDC: Eine Infektion wird als nosokomial bezeichnet, wenn der Infektionstag frühestens der Tag 3 des Krankenhausaufenthaltes ist. Dabei gilt der Aufnahmetag in das Krankenhaus als Tag 1 und der Tag mit dem ersten (spezifischen oder unspezifischen) Infektionszeichen als Infektionstag' ${ }^{1}$.

1 Diese Definition gilt nicht für postoperative Wundinfektionen. Die Definition für nosokomiale Infektionen in Bezug auf postoperative Wundinfektionen ist auf den Seiten des NRZ für nosokomiale Infektionen aufgeführt [33]

nicht als nosokomiale, sondern als mitgebrachte ${ }^{2}$ Infektionen klassifiziert.

Die Diagnose einer spezifischen nosokomialen Infektion für die Surveillance erfordert die Erfüllung einer spezifischen Definition. Dabei werden nicht alle, sondern nur ausgewählte nosokomiale Infektionen in umschriebenen $\mathrm{Pa}$ tientengruppen erfasst sowie nur solche, auf die die jeweilige Surveillance-Definition tatsächlich zutrifft. Definitionen

\footnotetext{
2 In einer anderen (ambulanten oder stationären) Gesundheitseinrichtung oder außerhalb von Gesundheitseinrichtungen erworben (engl. "community-acquired").
}

zur Surveillance von nosokomialen Infektion sind nicht für die klinische Entscheidungsfindung am Patientenbett entwickelt worden oder geeignet. Ein gutes Beispiel hierfür sind die gefäßkatheterassoziierten Blutstrominfektionen $\left(\mathrm{CABSI}^{3}\right)$. Die epidemiologisch orientierten Definitionen für nosokomiale Infektionen beweisen nicht, dass der Gefäßkatheter die Quelle der Blutstrominfektion ist. Trotzdem ließ sich in zahlreichen Studien durch gezielte Interventionen (Präventionsbündel) die Infektionsrate (CABSI pro 1000 Katheter-Anwendungstage) nachhaltig senken.

Im Einzelfall kann es sinnvoll sein, andere als die o.g. Definitionen anzuwenden. $\mathrm{Zu}$ beachten ist weiterhin, dass bestimmte nosokomiale Infektionen mit sehr kurzer oder langer Inkubationszeit (z.B. Norovirusinfektionen, Virushepatitis) mit den CDC- bzw. ECDC-Definitionen nicht adäquat erfasst werden können. Bei Vergleich mit den nationalen Referenzdaten können aktuell nur die KISS-Definitionen angewendet werden.

\footnotetext{
3 Aus dem Englischen: catheter-associated bloodstream infection. Im amerikanischen Sprachgebrauch auch CLABSI für ",central lineassociated BSI".
}

\subsection{Surveillance-Personal}

Es hat sich gezeigt, dass mit der Durchführung der Surveillance am besten das Hygieneteam des Krankenhauses betraut werden sollte. Das Hygienefachpersonal sollte für diese aktive Surveillance epidemiologisch weitergebildet sein. Es ist im Hinblick auf die konsequente Anwendung von Surveillance-Definitionen gewöhnlich objektiver als das ärztliche und pflegerische Personal der Stationen, da es nicht unmittelbar in die Behandlung einbezogen ist. Die Surveillance von nosokomialen Infektionen gehört zum originären Aufgabenspektrum der Hygienefachkräfte [38].

Auch Stationsärzte oder hygienebeauftragte Ärzte bzw. Ärzte in Einrichtungen für ambulantes Operieren können bei Beachtung der Definitionen nosokomialer Infektionen die Surveillance durchführen. In der Literatur wurde beschrieben, dass die sogenannte passive Surveillance durch das Stations- oder Abteilungspersonal zu mangelnder Sensitivität der Diagnosestellung von nosokomialen Infektionen führte [39-41].

Wenn die Surveillance durch Hygienefachkräfte erfolgt, sollte regelmäßig ein Austausch zwischen den behandelnden Ärzten (auch hygienebeauftragte Ärzte) und dem Hygienefachpersonal über die 
Tab. 3 Beispiele für Indikatoren für verschiedene nosokomiale Infektionen

\begin{tabular}{|c|c|}
\hline Nosokomiale Infektion & Indikatoren zur Fallfindung \\
\hline $\begin{array}{l}\text { Durch Labor bestätigte primäre } \\
\text { Blutstrominfektion }\end{array}$ & $\begin{array}{l}\text { Blutkulturbefunde, Fieber, Antibiotikatherapie, Wechsel des } \\
\text { zentralen Gefäßkatheters }\end{array}$ \\
\hline Pneumonie & $\begin{array}{l}\text { Mikrobiologische Befunde von bronchoalveolärer Lavage oder } \\
\text { Trachealsekret, Fieber, Röntgen-Thorax, bronchoskopische } \\
\text { Untersuchungen, Antibiotikagabe }\end{array}$ \\
\hline Harnwegsinfektionen & $\begin{array}{l}\text { Mikrobiologische Befunde, Fieber, Antibiotikagabe, Wechsel } \\
\text { des Harnwegskatheters }\end{array}$ \\
\hline Postoperative Wundinfektionen & $\begin{array}{l}\text { Diagnose des Arztes, mikrobiologische Ergebnisse von } \\
\text { Wundabstrichen oder Drainageflüssigkeit, Fieber, Antibiotika- } \\
\text { gabe, Revisionsoperationen, Wiederaufnahme eines Patienten }\end{array}$ \\
\hline C. difficile-Infektion & $\begin{array}{l}\text { Mikrobiologische Befunde, Diarrhö, CDI-spezifische Antibioti- } \\
\text { katherapie }\end{array}$ \\
\hline
\end{tabular}

Tab. 4 Unterschiedliche Informationen zu den Bezugsgrößen (Nennerdaten)

\begin{tabular}{|c|c|c|c|}
\hline Kategorie & Erläuterung & $\begin{array}{l}\text { Vorteile/ } \\
\text { Nachteile }\end{array}$ & Beispiele \\
\hline Patientenbezogen & $\begin{array}{l}\text { Für jeden einzel- } \\
\text { nen Patienten } \\
\text { werden Nenner- } \\
\text { daten erhoben } \\
\text { (z.B. ZVK-Tage, } \\
\text { Harnwegska- } \\
\text { theter-Tage, } \\
\text { Beatmungstage). }\end{array}$ & $\begin{array}{l}\text { Gute Möglichkeit } \\
\text { für die Risikoad- } \\
\text { justierung, aber } \\
\text { hoher zeitlicher } \\
\text { Aufwand für die } \\
\text { Erfassung der } \\
\text { „Device“-Tage, } \\
\text { sofern nicht digital } \\
\text { verfügbar }\end{array}$ & $\begin{array}{l}\text { Postoperative } \\
\text { Wundinfektions- } \\
\text { raten pro } 100 \\
\text { OPs einer Art } \\
\text { unter Berück- } \\
\text { sichtigung des } \\
\text { Wundinfektions- } \\
\text { risikos (NNIS- } \\
\text { Index) }\end{array}$ \\
\hline $\begin{array}{l}\text { Stationsbezogen/ } \\
\text { Abteilungsbezogen }\end{array}$ & $\begin{array}{l}\text { Nur summari- } \\
\text { sche Erfassung } \\
\text { der "Device“-Tage } \\
\text { für die Gesamt- } \\
\text { gruppe (Station/ } \\
\text { Abteilung) }\end{array}$ & $\begin{array}{l}\text { Eingeschränkte } \\
\text { Möglichkeit der } \\
\text { Risikoadjustie- } \\
\text { rung, mittlerer } \\
\text { zeitlicher Aufwand }\end{array}$ & $\begin{array}{l}\text { Beatmungsasso- } \\
\text { ziierte Pneu- } \\
\text { monieraten pro } \\
1000 \text { "Device“- } \\
\text { Tage }\end{array}$ \\
\hline $\begin{array}{l}\text { Krankenhausbezogen/Einrichtungs- } \\
\text { bezogen }\end{array}$ & $\begin{array}{l}\text { Nur summarische } \\
\text { Erfassung der } \\
\text { "Device"-Tage für } \\
\text { die Gesamtgrup- } \\
\text { pe (Krankenhaus) }\end{array}$ & $\begin{array}{l}\text { Kaum Möglichkeit } \\
\text { der Risikoadjus- } \\
\text { tierung, geringer } \\
\text { zeitlicher Aufwand }\end{array}$ & $\begin{array}{l}\text { Nosokomiale } C \text {. } \\
\text { difficile-Infekti- } \\
\text { onen pro } 1000 \\
\text { Patiententage }\end{array}$ \\
\hline
\end{tabular}

für die Surveillance als nosokomial infiziert eingestuften Patienten erfolgen, vor allem um die spätere Akzeptanz der ermittelten Infektionsraten zu erreichen.

Für die KISS-Teilnahme ist die Teilnahme an einem Einführungskurs zum Erlernen und Trainieren der KISS-Definitionen erforderlich sowie regelmäßige Wiederholungen dieses Trainings, damit es nicht zu einer unterschiedlichen Interpretation der Definitionen kommt [42]. Mit Hilfe dieses Trainings konnte im KISS in der Überprüfung anhand von Fallbeispielen eine mittlere Sensitivität von $86 \%$ und eine Spezifität von $95 \%$ erreicht werden [42].

\subsection{Erkennung von Infektionsfällen (Zählerdaten) mit Hilfe von Indikatoren}

Hygienefachpersonal soll die Surveillance nicht ausschließlich auf der Basis der Informationen des Krankenhaus-Informationssystems durchführen, sondern hierfür regelmäßig auf den Stationen anwesend sein. Die regelmäßige Anwesenheit des Hygienefachpersonals allein kann einen wichtigen infektionspräventiven Effekt auslösen (Hawthorne-Effekt), weil dadurch die Aufmerksamkeit für das Thema gesteigert wird bzw. auf einem hohen Niveau gehalten werden kann.
Im Sinne der Effektivität der Surveillance und um die Identifizierung von Patienten mit möglichen nosokomialen Infektionen zu erleichtern, können Indikatoren verwendet werden. • Tab. 3 zeigt mögliche Indikatoren für verschiedene Infektionsarten. Eine enge Zusammenarbeit mit dem Stations-/Abteilungspersonal ist Voraussetzung, um zusätzliche Hinweise auf möglicherweise nosokomial infizierte Patienten zu erhalten (hygienebeauftragtes Pflegepersonal, hygienebeauftragte Ärzte).

Die Erhebung der o.g. Indikatoren wird wesentlich erleichtert, wenn für die Erfassung zentrale Informationen schon grundsätzlich gut erkennbar in den Patientenakten vermerkt sind. Hierzu gehören z. B. Diagnosen, Operationen/Eingriffe und Fremdkörper (Katheter, Tubus etc.) sowie Antibiotikatherapie, mikrobiologische Befunde, Entzündungszeichen, Durchfall/ Erbrechen und Fieber [43]. Bei Vorliegen von Indikatoren muss im nachfolgenden Schritt überprüft werden, ob eine nosokomiale Infektion nach den festgelegten Kriterien (z. B. KISS-Definitionen) vorliegt.

Sofern die Indikatoren mit Hilfe des Krankenhaus-Informationssystems bereitgestellt werden, bezeichnet man dieses Vorgehen auch als semiautomatische Surveillance. Bei der Weiterentwicklung von Krankenhaus-Informationssystemen sollten die o.g. Parameter nach Möglichkeit integriert werden, um eine semiautomatische Surveillance zu ermöglichen.

Selbstverständlich können nosokomiale Infektionen auch nach der Entlassung aus dem Krankenhaus bzw. einer Einrichtung für ambulantes Operieren auftreten, für das/die die Surveillance durchgeführt wird. Solche nosokomialen Infektionen sollen registriert werden, wenn sie bekannt werden. Allerdings ist deren systematische Erfassung unter den heutigen Bedingungen in der Regel sehr aufwendig und kann durch viele Einrichtungen nicht geleistet werden. Deshalb können diese Infektionen bei den vergleichenden Analysen (z.B. in KISS) nicht berücksichtigt werden.

\subsection{Bestimmung der Bezugsdaten (Nennerdaten)}

Die Surveillance nosokomialer Infektionen ist eine gesetzliche Verpflichtung, die der Infektionsprävention dient und sich 


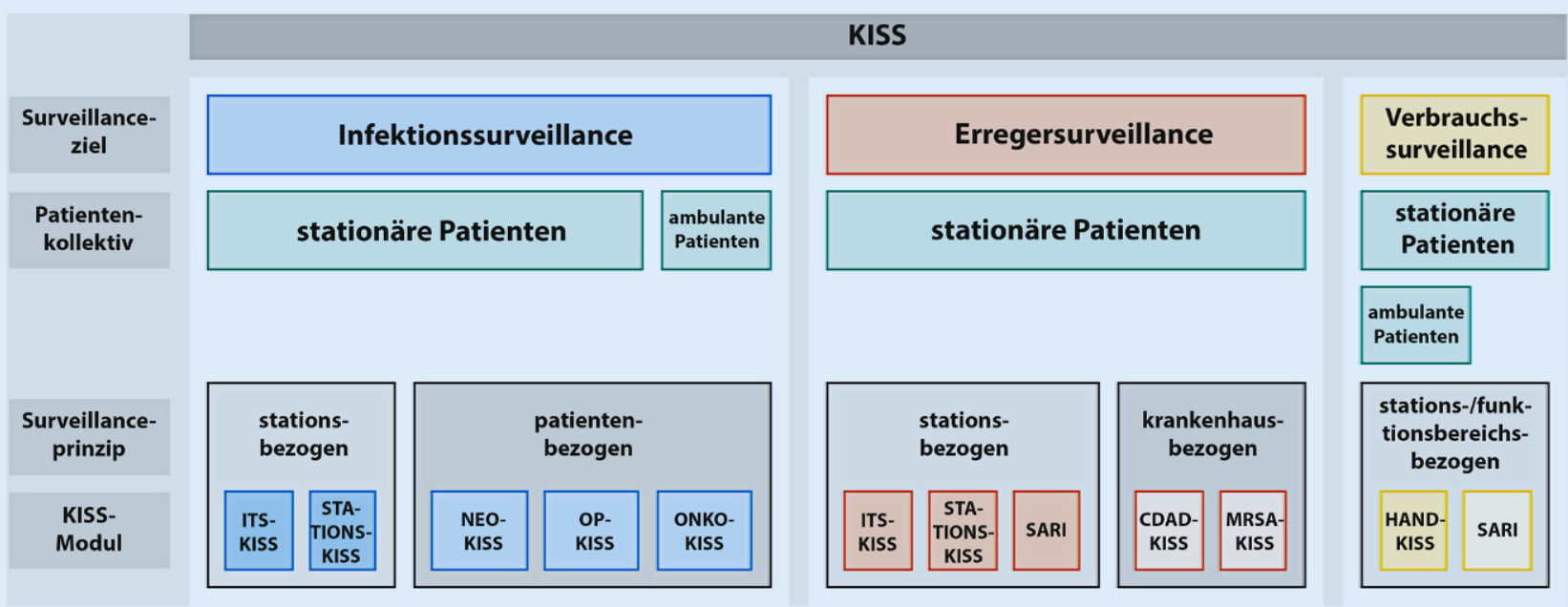

Abb. 1 A Modulübersicht: Krankenhaus-Infektions-Surveillance-System, Stand 2018

auf die Erhebung notwendiger Parameter konzentriert. Deshalb ist es bei vielen Surveillance-Ansätzen nicht notwendig, umfangreiche Informationen über alle Patienten unter Surveillance zu erfassen, sondern es reichen oft zusammenfassende Daten zu den wichtigsten Risikofaktoren für die Entwicklung von nosokomialen Infektionen in dieser Population aus, um eine günstige Zeitaufwand-NutzenRelation zu ermöglichen.

Es ist möglich, die Bezugsdaten patientenbezogen, stationsbezogen oder einrichtungsbezogen zu erheben ( $\bullet$ Tab. 4). Bei der patientenbezogenen Methode werden die Nennerdaten (z. B. „Device“Tage) individuell für jeden Patienten aufgezeichnet, bei der stationsbezogenen Surveillance nur summarisch für die gesamte Station bzw. bei der einrichtungsbezogenen Surveillance summarisch für das gesamte Krankenhaus bzw. für die gesamte Einrichtung. Auf den Internet-Seiten des NRZ für Surveillance von nosokomialen Infektionen wird die Berechnung der Nennerdaten detailliert dargestellt [44].

Bei bestimmten seltenen nosokomialen Infektionen ist es zudem sinnvoll, die Infektionsfälle zu erfassen ohne sie auf Nennerdaten zu beziehen, sondern den Bezug zur Abteilung oder zum Krankenhaus/zur Einrichtung als Ganzes herzustellen. Beispiele hierfür sind Legionellosen oder invasive Aspergillosen.

\subsection{Surveillance-Protokolle}

Surveillance-Protokolle beschreiben die Methode der Surveillance, also z. B. die Erfassung der Zähler- und Nennerdaten und die Bestimmung der Infektionsraten für verschiedene Infektionsarten und Patientengruppen. Durch die Anwendung einheitlicher Surveillance-Protokolle können annähernd vergleichbare Surveillance-Daten erzeugt werden. Surveillance-Protokolle von relevanten nosokomialen Infektionen, sowie eine Anleitung zur Berechnung der Infektionsraten und zum Vergleich mit Referenzdaten sind auf den Internetseiten des NRZ für Surveillance von nosokomialen Infektionen hinterlegt [33]. Bei Anwendung individueller Surveillance-Methoden im eigenen Bereich helfen Surveillance-Protokolle die einheitliche Durchführung sicherzustellen, auch über längere Zeitabschnitte oder bei Wechsel der Verantwortlichkeiten.

Bei Beteiligung an KISS ist die Beachtung der KISS-Protokolle verpflichtend, um Vergleiche mit den Referenzdaten gewährleisten zu können. Um die wichtigsten Risikofaktoren der Patienten zu berücksichtigen, wird zusätzlich versucht, durch Stratifizierung und Standardisierung möglichst gut vergleichbare Referenzdaten zu bilden. Stratifizierung bedeutet in diesem Zusammenhang, dass separate Infektionsraten für Patienten in unterschiedlichen Risikokategorien gebildet werden (z.B. Stratifizierung nach
Geburtsgewicht bei Frühgeborenen unter $1500 \mathrm{~g}$ ). Unter Standardisierung ist die Bezugnahme auf den Umfang der Exposition zu verstehen (z. B. Tage mit Gefäßkatheter oder unter Beatmung). Für verschiedene Risikogruppen können auch standardisierte Infektionskennzahlen (SIK) berechnet werden. Dabei werden für die Berechnung der erwarteten Infektionen die Ergebnisse aller am Surveillance-System teilnehmenden Einrichtungen zugrunde gelegt.

$S I K=\frac{\begin{array}{c}\text { beobachtete nosokomiale } \\ \text { Infektionen }\end{array}}{\begin{array}{c}\text { erwartete nosokomiale Infektionen } \\ \text { (nach Risikostruktur stratifiziert) }\end{array}}$

\subsection{Orientierung an nationalen Daten}

Für die gezielte Identifikation von Problemen und die Einführung von zusätzlichen Präventionsmaßnahmen bzw. Überprüfung der Umsetzung der bisherigen Präventionsmaßnahmen ist es nützlich, wenn für die Interpretation der eigenen Surveillance-Ergebnisse Referenzdaten zur Verfügung stehen. In den USA werden daher zum Vergleich Daten des National Healthcare Safety Network (NHSN) herangezogen [45]. Viele europäische Länder haben in den neunziger Jahren vergleichbare Systeme entwickelt und durch die ECDC wird inzwischen eine europaweite Surveillance für verschiedene nosokomiale Infektionen organisiert [46]. 
Tab. 5 Übersicht über die gemäß $§ 23$ Abs. 4 in Verbindung mit $\S 4$ Abs. 2 Nr. 2 IfSG zu erhebenden nosokomialen Infektionen, Stand 2013 [2]

Je nach den einrichtungsspezifischen Erfordernissen (d.h. entsprechend den nachvollziehbar identifizierten Risikobereichen) sind aus der folgenden Liste die in der jeweiligen Einrichtung (Krankenhaus bzw. Einrichtung für ambulantes Operieren) für die Erfassung und Bewertung jeweils geeigneten und angemessenen aussagekräftigen nosokomialen Infektionen auszuwählen und festzulegen:

- Postoperative Wundinfektionen (anhand geeigneter Indikatoroperationen)

- Katheterassoziierte Septikämien

- Beatmungsassoziierte Pneumonien

- Katheterassoziierte Harnwegsinfektionen

Nosokomiale Diarrhöen durch C. difficile (CDAD) sollen in allen Bereichen eines Krankenhauses erhoben werden.

In Deutschland wurde das Krankenhaus-Infektions-Surveillance-System (KISS) ab 1996 durch das NRZ für Surveillance von nosokomialen Infektionen aufgebaut [47]. Die Teilnahme am KISS ist freiwillig, aktuell nehmen ca.1400 Kliniken an verschiedenen Modulen teil. Das KISS hat für die Surveillance der wichtigsten nosokomialen Infektionen bei bestimmten Risikogruppen verschiedene Surveillance-Module entwickelt und liefert entsprechende Surveillance-Protokolle und Referenzdaten (- Abb. 1). Die Surveillance-Module wurden in erster Linie für Krankenhäuser entwickelt, für den ambulanten Bereich werden in Zukunft weitere Module entwickelt.

Die durch das KISS erzeugten nationalen Referenzdaten können zusätzlich auch für die Beurteilung der epidemiologischen Situation in ausgewählten Bereichen herangezogen werden.

\subsection{Unterstützung durch die Informatik und das Controlling}

Fachexperten aus den Bereichen Controlling und Informatik eines Krankenhauses können einen wichtigen Beitrag zur Vereinfachung und damit zur Effizienzsteigerung bei der Surveillance leisten. Je besser die Unterstützung von dieser Seite ist, desto mehr Bereiche können in die Surveillance einbezogen werden bzw. Ressourcen für andere Aufgaben in der Infektionsprävention freigesetzt werden.

Mit Hilfe der semiautomatischen Surveillance kann der Zeitaufwand für die Identifizierung von möglicherweise nosokomi- al infizierten Patienten deutlich reduziert werden, ohne zu einer Reduktion der Sensitivität zu führen [48-51].

Für eine automatische Surveillance von nosokomialen Infektionen, bei der die Infektionsfälle nur durch einen Computeralgorithmus auf der Basis des KrankenhausInformationssystems generiert werden, sind in den meisten deutschen Krankenhäusern die Voraussetzungen noch nicht gegeben [52]. Darüber hinaus sollten die notwendigen Nennerdaten so bereitgestellt werden, dass sie vom Hygienefachpersonal ohne weitere Zwischenschritte übernommen werden können. Das bezieht sich z. B. auf die für die Surveillance ausgewählten OP-Arten (inklusive Risikofaktoren der Patienten, NNIS-Index) oder die Tage, an denen bei den Intensivpatienten bestimmte „devices“ (zentrale Gefäßkatheter, invasive Beatmung, Harnwegskatheter) vorlagen.

\section{Auswahl der Infektionsarten und Bereiche für die Surveillance in der eigenen Einrichtung}

Unter Surveillance-Experten existiert international große Übereinstimmung, dass eine krankenhausweite Erfassung aller nosokomialen Infektionen weder sinnvoll noch kosteneffektiv ist. Deshalb sollte eine Konzentration auf die Bereiche im Krankenhaus erfolgen, wo besonders viele Risikopatienten für nosokomiale Infektionen behandelt werden (z.B. Intensivpatienten, Frühgeborene, hämatologisch-onkologische Patienten). Außerdem sollten insbesondere die nosokomialen Infektionen erfasst werden, die einerseits häufig genug auftreten, sodass sinnvolle Infektionsraten berechnet werden können, und/ oder die andererseits auch eine hohe Relevanz im Hinblick auf Morbidität, Letalität und Kosten haben (z.B. BSI, Pneumonien, postoperative Wundinfektionen, C. difficile-Infektionen). Auch das jeweilige Präventionspotential spielt eine Rolle.

\subsection{Auszuwählende Bereiche}

Neben den oben genannten Risikobereichen eines Krankenhauses kann es auch in anderen Bereichen sinnvoll sein, eine Surveillance durchzuführen, vor allem wenn es dort wiederholt zu Häufungen von nosokomialen Infektionen gekommen ist. Querschnittsuntersuchungen (Prävalenz) können Hinweise auf mögliche Risikobereiche geben und erhöhen gleichzeitig die Aufmerksamkeit für das Thema in einem bestimmten Bereich.

Der festzulegende Bereich kann den Ort der Surveillance (Station, Abteilung) und die Art der nosokomialen Infektion betreffen, aber auch andere für die Infektionsprävention relevante Parameter. So kann der Fokus beispielswiese auch auf die Anwendungsraten von Harnwegskathetern oder Gefäßkathetern gelegt werden, weil hier möglicherweise ein weiterer wichtiger Ansatzpunkt für die Prävention von nosokomialen Infektionen gegeben ist.

\subsection{Auszuwählende Infektionen}

Als nosokomiale Infektionen, deren Auftreten nach $\$ 23$ Abs. 4 Satz 1 IfSG fortlaufend in einer gesonderten Niederschrift aufzuzeichnen und zu bewerten sind, damit sachgerechte Schlussfolgerungen hinsichtlich erforderlicher Präventionsmaßnahmen gezogen werden können, wurden die in - Tab. 5 genannten festgelegt. Es ist $\mathrm{zu}$ beachten, dass diese Liste entsprechend den epidemiologischen Erfordernissen vom RKI fortgeschrieben wird [2].

Diese Verpflichtung soll die Einrichtungen in die Lage versetzen, eigene Schwächen im Hygienemanagement zu erkennen und gegebenenfalls die notwendigen Hygienemaßnahmen, einschließlich der Schulung des Personals bzw. der kritischen Bewertung des Antibiotikaeinsatzes, $\mathrm{zu}$ verstärken oder $\mathrm{zu}$ etablieren 
bzw. der Verbreitung der betreffenden Erreger möglichst schnell Einhalt zu gebieten. Bei der Ausgestaltung dieser Vorschriften zur Krankenhaushygiene war seitens des Gesetzgebers bedacht worden, dass regelmäßige epidemiologische Untersuchungen je nach ihrem Umfang mit einem Zeitaufwand verbunden sind und die Surveillance sich deshalb auf das erforderliche und sinnvolle Maß beschränken sollte [53]. Entscheidend sind die nachvollziehbare Identifizierung der Risikobereiche und das Präventionspotenzial bei den ausgewählten Infektionen.

Damit ermöglicht das RKI eine Auswahl der Infektionsarten, die für die eigene Einrichtung besonders relevant sind. In manchen Einrichtungen können aber durchaus auch andere Endpunkte eine hohe Bedeutung haben, z. B. in neurochirurgischen Intensivstationen ventrikeldrainageassoziierte Meningitis/Ventrikulitis oder in operativen Abteilungen postoperative Pneumonien nach bestimmten OPArten.

Bestimmte Einrichtungen haben auf Grund der geringen Anzahl nosokomialer Infektionen ein so geringes Präventionspotential, dass eine Surveillance nicht gerechtfertigt ist (z. B. Psychosomatik). Die Aufwand-Nutzen-Relation (Zeitaufwand für die Surveillance versus Präventionspotential bei den nosokomialen Infektionen) sollte bei der Auswahl der Infektionsarten immer beachtet werden.

\section{Interpretation von Surveillance-Daten}

Bei der Interpretation von SurveillanceDaten muss im Vordergrund stehen, eine Verbesserung der Situation in der jeweiligen Abteilung zu erreichen, d.h. es muss auf geeignete Ansatzpunkte für das Feedback an die Station/Abteilung geachtet werden. Dabei ist der Verlauf der Infektionsraten über die Zeit relevant, das Auftreten bestimmter Erreger, aber natürlich auch der Vergleich mit anderen Abteilungen/Stationen mit Hilfe der Referenzdaten von KISS. Auch wenn z. B. KISS regelmäßig Konfidenzintervalle für die Infektionsraten liefert, heißt das nicht, dass erst reagiert werden sollte, wenn ein signifikanter „outlier"-Status einer Abteilung/ Station vorliegt oder die Infektionsraten oberhalb der 75. Perzentile in der Verteilung der Infektionsraten liegen. Möglicherweise hat eine Abteilung/Station auch nur deshalb vergleichsweise günstige Infektionsraten, weil viele nosokomiale Infektionen nicht diagnostiziert werden.

Für die Interpretation der Infektionsraten ist auch die Analyse der zugrundeliegenden Einzelfälle relevant (z. B. individuelle Risikofaktoren der betroffenen Patienten für nosokomiale Infektionen).

Bei der Interpretation von Infektionsraten im Vergleich zu den Referenzdaten von KISS sind folgende Faktoren zu beachten:

- Länge der Surveillance-Periode. Bei zu kurzen Perioden könnten hohe oder niedrige Infektionsraten durch zufällige Ereignisse bedingt sein.

- Umfang der mikrobiologischen Diagnostik bzw. anderer diagnostischer Verfahren.

Beispielsweise gibt es große Unterschiede in den Blutkulturentnahmeraten zwischen den Intensivstationen, die auch einen Einfluss auf die Rate der Blutstrominfektionen haben.

- Erfahrung, Ausbildung, Motivation und Genauigkeit des SurveillancePersonals (Sensitivität und Spezifität der Erfassung). Beispielsweise verlangt KISS für alle mit der Surveillance betrauten Personen die Teilnahme an einem Einführungskurs, um die Anwendung der KISS-Definitionen zu trainieren. Trotzdem gibt es Unterschiede zwischen den mit der Surveillance befassten Personen, die auch einen Einfluss auf die Infektionsraten haben können [42, 54].

- Trotz Anwendung verschiedener Verfahren zur Berücksichtigung der Erkrankungsschwere der Patienten ist es nicht möglich, die entsprechenden Unterschiede der Patientenpopulationen in verschiedenen Krankenhäusern vollumfänglich zu berücksichtigen.

- Weitere Faktoren (z. B. regionale Unterschiede im Vorkommen bestimmter Erregerstämme).

Bei der Interpretation von SurveillanceDaten im Rahmen der internen Qualitätssicherung können und sollten solche Faktoren berücksichtigt werden.

\section{Hinweise zum Feedback von Surveillance-Daten}

Surveillance-Ergebnisse sollen regelmäßig (z. B. ein- oder zweimal pro Jahr und anlassbezogen) an die jeweiligen Stationen/ Abteilungen/ambulanten Einrichtungen übermittelt werden. Die Ergebnisse sollten schriftlich an den Leiter der Abteilung bzw. Einrichtung gesendet werden, aber auch im Team der Station/Abteilung/Einrichtung präsentiert werden (möglichst ärttliches und pflegerisches Personal gemeinsam), um eine Diskussion zur Optimierung der Infektionsprävention anzuregen. Dabei sollten möglichst viele Angehörige des Stations-/Abteilungspersonals bzw. der ambulanten Einrichtung einbezogen werden. Die Daten sollten so präsentiert werden, dass es einfach ist, die Entwicklung über die Zeit und die eigene Position im Vergleich zu anderen Stationen/Abteilungen/ambulanten Einrichtungen zu identifizieren (z.B. durch Präsentation geeigneter Abbildungen). Gegebenenfalls sollten einzelne Kasuistiken für die Datenvorstellung aufgearbeitet werden. Wenn nötig sollen Maßnahmen festgelegt und ihre Umsetzung kontrolliert werden.

Bei anhaltend hohen Infektionsraten auf einer Station/in einer Abteilung oder ambulanten Einrichtung wird folgendes Vorgehen empfohlen:

- Evaluation der Infektionsfälle, um mit Hilfe der Durchsicht der Patientenakten Hinweise auf Verbesserungspotential zu erhalten

- Hospitation, um infektionsrelevante Abläufe und Verhaltensweisen zu beurteilen

- Erarbeitung eines stations-/abteilungs-/einrichtungsspezifischen Maßnahmenbündels in enger Zusammenarbeit mit der betroffenen Station/ Abteilung und ihrer Leitung

- Implementierung eines Maßnahmenbündels in Zusammenarbeit mit dem Abteilungs-/Stationspersonal bzw. mit dem Personal der Einrichtung

- Kontrolle der Implementierung

- Begleitende Evaluation durch weitere Surveillance

Selbstverständlich sollte auch untersucht werden, ob es nachvollziehbare Erklärun- 
gen für die erhöhten Infektionsraten gibt, die außerhalb des Präventionspotentials liegen (z. B. besondere Zusammensetzung der Patientengruppe).

\section{Evaluation der Surveillance in der eigenen Einrichtung}

Regelmäßig (z. B. einmal jährlich) sollen die im Rahmen der Surveillance eingesetzten Prozesse kritisch überprüft werden. Außerdem sollte man regelmäßig überprüfen, ob die Weiterführung der Surveillance in einer bestimmten Station/ Abteilung in der bisherigen Form sinnvoll ist. Beispielsweise könnte sie bei begrenzten Ressourcen in einer Station/Abteilung mit sehr günstigen Infektionsraten zugunsten einer anderen Abteilung unterbrochen werden.

Wenn sich in einer Abteilung mit vergleichsweise hohen Infektionsraten über längere Zeit kein Trend zur Verbesserung zeigt, soll überprüft werden, ob die bisherige Art des Feedbacks der Infektionsraten ausreichend und geeignet war, um Interventionen $\mathrm{zu}$ stimulieren und ob die Interventionen sinnvoll waren (siehe oben).

\section{Surveillance im Sinne des internen Qualitätsmanagements versus externe Qualitätssicherung}

Viele Patienten, auch in Deutschland, wünschen sich Qualitätsparameter, mit deren Hilfe sie die Anstrengungen eines Krankenhauses auf dem Gebiet der Infektionsprävention beurteilen können. Ausgewählte Infektionsraten aus einzelnen Risikobereichen sind dafür wenig geeignet, weil sie durch Laien schwer zu interpretieren sind. Hier sollte man sich besser an der entsprechenden Strukturqualität einer Einrichtung und den etablierten Prozessen auf dem Gebiet der Infektionsdiagnostik und-prävention sowie am Umfang und an der Qualität der Surveillance von nosokomialen Infektionen orientieren.

Anfang des Jahrtausends haben einige Länder die Teilnahme an nationalen oder regionalen Surveillance-Systemen für verschiedene nosokomiale Infektionen für verpflichtend erklärt und überwiegend auch eine Veröffentlichungspflicht der Surveillance-Daten eingeführt. Das gilt zum Beispiel in den USA (mit Unterschieden zwischen den Bundesstaaten) für katheterassoziierte Blutstrominfektionen, katheterassoziierte Harnwegsinfektionen und postoperative Wundinfektionen nach ausgewählten OP-Arten [53]. In England wurde die verpflichtende Surveillance inklusive Veröffentlichungspflicht für postoperative Wundinfektionen nach Hüft- und Knieendoprothesen eingeführt sowie für C. difficile-Infektionen $[55,56]$. In Frankreich existiert eine Verpflichtung zur Surveillance der postoperativen Wundinfektionen und zur Publikation der Daten [57], in Kanada für C. difficileInfektionen [58]. Obwohl die Veröffentlichungspflicht in diesen Ländern schon vor vielen Jahren eingeführt wurde, existieren bisher nur wenige Berichte über ihre Effekte auf die Raten nosokomialer Infektionen, mit widersprüchlichen Ergebnissen [58-61].

In Deutschland wurde durch den Gemeinsamen Bundesausschuss im Jahre 2005 eine verpflichtende Teilnahme an einem Infektions-Surveillance-System für Level 1 und 2 Perinatalzentren gefordert [62]. Die Perinatalzentren kommen dieser Forderung mehrheitlich durch Teilnahme am NEO-KISS (Surveillance-Modul für Frühgeborene auf Intensivstationen) nach. Eine Publikationspflicht der Infektionsraten ergibt sich daraus nicht.

Seit 01.01.2017 existiert im Rahmen des Qualitätssicherungsverfahrens „Vermeidung nosokomialer Infektionen postoperative Wundinfektionen (QSWI)“ der Richtlinie zur datengestützten einrichtungsübergreifenden Qualitätssicherung nach $\$ 136$ Abs. 1 Satz 1 Nr. 1 SGB V (bis zum 31.12.2019 Richtlinie zur einrichtungs- und sektoren-übergreifenden Qualitätssicherung) des Gemeinsamen Bundesausschusses die Verpflichtung zur Dokumentation stationärer Fälle, bei denen es sich um potentielle postoperative Wundinfektionen handelt. Die Dokumentationspflicht wird bei bestimmten ICD10- und OPS-Code-basierten Konstellationen ausgelöst. Dokumentiert wird das Vorliegen einer postoperativen Wundinfektion, die Wundtiefe und ob eine mikrobiologische Diagnostik durchgeführt wurde. Diese Datenerhebung ersetzt nicht die Vorgaben der Surveillance nach $\$ 23$ IfSG als Maßnahme des internen Qualitätsmanagements.

Häufig wird eine Veröffentlichungspflicht von Qualitätsparametern zur Infektionsprävention gefordert, um eine höhere Aufmerksamkeit für das Thema und zusätzliche Anstrengungen zur Infektionsprävention zu erreichen. Da Infektionsraten der „härteste“ Endpunkt der Qualitätssicherung auf diesem Gebiet sind, wird die Veröffentlichung der Infektionsraten der Einrichtungen gefordert.

Viele Experten sehen die Einführung einer verpflichtenden Surveillance in Bezug auf bestimmte Infektionsarten und Bereiche sowie die Publikation der nosokomialen Infektionsraten kritisch [52, 63, 64]. Ein wichtiges Argument gegen die verpflichtende Surveillance ist die Tatsache, dass die Surveillance einen erheblichen Zeitaufwand erfordert und sie nur dann Kosten-Nutzen-effektiv sein kann, wenn Infektionsprobleme vorliegen. Deshalb sollten die Krankenhäuser selbst festlegen können, in welchen Bereichen und in Bezug auf welche Infektionsarten sie eine Surveillance von nosokomialen Infektionen durchführen. Gegen die Publikationspflicht der Infektionsraten sprechen vor allem folgende Argumente:

- Abhängig vom Diagnostikumfang: Surveillance-Daten sind in erheblichem Maße vom Ausmaß und der Qualität der Diagnostik, insbesondere der mikrobiologischen Diagnostik abhängig. Bei einer konsequent leitlinienkonformen infektiologischen Diagnostik könnte der Eindruck entstehen, dass die Einrichtung vergleichsweise hohe Raten nosokomialer Infektionen hat [65].

- Beeinflussung von individuellen Erfassungsmerkmalen: SurveillanceDaten sind von der Erfahrung, Ausbildung und Genauigkeit des Surveillance-Personals (Sensitivität und Spezifität der Erfassung) bei der Anwendung der Definitionen für nosokomiale Infektionen abhängig. Eine hohe Sensitivität bei der Detektion nosokomialer Infektionen durch geschultes Personal führt zu signifikant höheren Infektionsraten [42].

- Eingeschränkte Vergleichbarkeit: Ein hoher Anteil von Risikopatienten unter den Patienten unter Surveillance 


\begin{tabular}{|c|c|}
\hline \multicolumn{2}{|c|}{ Abkürzungen } \\
\hline$B S I$ & Blutstrominfektion \\
\hline CABSI & $\begin{array}{l}\text { catheter-associated } \\
\text { bloodstream infection, } \\
\text { gefäßkatheterassoziierte } \\
\text { Blutstrominfektion }\end{array}$ \\
\hline$C D A D$ & $\begin{array}{l}\text { Clostridioides difficile- } \\
\text { assoziierte Diarrhö }\end{array}$ \\
\hline$C D C$ & $\begin{array}{l}\text { Centers for Disease Control and } \\
\text { Prevention }\end{array}$ \\
\hline$C D I$ & Clostridioides difficile-Infektion \\
\hline$E C D C$ & $\begin{array}{l}\text { European Centre for Disease } \\
\text { Prevention and Control }\end{array}$ \\
\hline IfSG & Infektionsschutzgesetz \\
\hline IQTIG & $\begin{array}{l}\text { Institut für Qualitätssicherung } \\
\text { und Transparenz im Gesund- } \\
\text { heitswesen }\end{array}$ \\
\hline KISS & $\begin{array}{l}\text { Krankenhaus-Infektions- } \\
\text { Surveillance-System }\end{array}$ \\
\hline KRINKO & $\begin{array}{l}\text { Kommission für Krankenhaus- } \\
\text { hygiene und Infektionsprä- } \\
\text { vention }\end{array}$ \\
\hline NEO-KISS & $\begin{array}{l}\text { Krankenhaus-Infektions-Surveil- } \\
\text { lance-System für Frühgeborene } \\
\text { auf Intensivstationen }\end{array}$ \\
\hline NHSN & $\begin{array}{l}\text { National Healthcare Safety } \\
\text { Network }\end{array}$ \\
\hline NNIS & $\begin{array}{l}\text { National Nosocomial Infections } \\
\text { Surveillance }\end{array}$ \\
\hline$N R Z$ & Nationales Referenzzentrum \\
\hline$O P$ & Operation \\
\hline QSWI & $\begin{array}{l}\text { Qualitätssicherungsverfahren } \\
\text { Vermeidung nosokomialer } \\
\text { Infektionen - postoperative } \\
\text { Wundinfektionen }\end{array}$ \\
\hline RKI & Robert Koch-Institut \\
\hline SENIC & $\begin{array}{l}\text { Study on the Efficacy of } \\
\text { Nosocomial Infection Control }\end{array}$ \\
\hline SIK & $\begin{array}{l}\text { Standardisierte Infektionskenn- } \\
\text { zahlen }\end{array}$ \\
\hline WHO & $\begin{array}{l}\text { World Health Organization, } \\
\text { Weltgesundheitsorganisation }\end{array}$ \\
\hline ZVK & Zentraler Venenkatheter \\
\hline
\end{tabular}

führt in der Regel zu höheren nosokomialen Infektionsraten, weil die üblichen Methoden der Adjustierung von Surveillance-Arten das Risikoprofil der Patienten nur bedingt abbilden können.

Mit der Publikationspflicht können Tendenzen zur Manipulation von Surveillance-Daten erzeugt werden, die die Qua- lität der Surveillance-Daten an sich und damit ihre effektive Anwendung zur Infektionsprävention beeinträchtigen. Hinzu kommt, dass durch den starken Fokus auf die ausgewählten Infektionsarten bzw. -bereiche eine übermäßige Konzentration der Präventionsanstrengungen auf diese Infektionen bzw. Bereiche zustande kommen kann („Tunnelblick“).

In manchen Ländern wurde über die Verpflichtung zur Publikation der Surveillance-Daten hinaus eingeführt, dass die Krankenhäuser bei Auftreten von bestimmten nosokomialen Infektionen monetäre Abzüge erhalten bzw. die Zusatzkosten für die Behandlung der Infektionen nicht mehr vergütet werden. Beispielsweise wurden in den USA solche Regelungen für ZVK-assoziierte Blutstrominfektionen, katheterassoziierte Harnwegsinfektionen und postoperative Wundinfektionen bei bestimmten OP-Arten eingeführt $[66,67]$. Teilweise wurde berichtet, dass daraufhin die Diagnostik reduziert wurde, um weniger Infektionen zu erfassen [68]. Mögliche Tendenzen zur Manipulation von Surveillance-Daten sind unter diesen Bedingungen (pay for performance) in noch höherem Maße gegeben.

\section{Empfehlungen}

\section{Die Kommission empfiehlt:}

- die Durchführung einer Surveillance von nosokomialen Infektionen nach den Vorgaben des IfSG und den Festlegungen des Robert Koch-Institutes (Kat. IV);

- die Auswahl der zu erfassenden nosokomialen Infektionen für definierte Risikobereiche in der Hygienekommission zu treffen (ohne Kategorie);

- eine bevorzugte Anwendung der Definitionen und Surveillance-Protokolle von KISS, um sich an Vergleichsdaten orientieren zu können (Kat. II);

- Training der für die Surveillance verantwortlichen Mitarbeiter in der Anwendung der Definitionen anhand von konkreten klinischen Beispielen oder Fallbeispielen zur Verbesserung der Sensitivität und Spezifität der Infektionserfassung (Kat. II);

- eine umfangreiche Unterstützung der Surveillance durch das Controlling und den Informationstechnologie-
Bereich des Krankenhauses, um eine hohe Effizienz zu erreichen (z. B. Entwicklung semiautomatischer Systeme) (ohne Kategorie);

- die Einbeziehung aller in die Behandlung involvierten Mitarbeiter in die Kommunikation von SurveillanceDaten sowie die Präsentation der Daten in einer Form, die deren Analyse und damit die ggf. abzuleitenden Interventionen fördert (ohne Kategorie);

- die regelmäßige Evaluierung und ggf. Anpassung des Surveillance-Programmes (ohne Kategorie).

Interessenkonflikt. Diese Empfehlung wurde ehrenamtlich und ohne Einflussnahme kommerzieller Interessengruppen im Auftrag der Kommission für Krankenhaushygiene und Infektionsprävention erarbeitet von Prof. Dr. Petra Gastmeier (Leiterin der Arbeitsgruppe und des NRZ für Surveillance von nosokomialen Infektionen), PD. Dr. Christian Brandt, Prof. Dr. Christine Geffers (NRZ für Surveillance von nosokomialen Infektionen), Prof. Dr. Simone Scheithauer sowie Prof. Dr. Constanze Wendt. Vom RKI waren beteiligt: Dr. Tim Eckmanns und Dr. Anna Stoliaroff-Pépin. Die Empfehlung wurde durch die Arbeitsgruppe vorbereitet und nach ausführlicher Diskussion in der Kommission abgestimmt.

\section{Literatur}

1. Langmuir A (1963) The surveillance of communicable diseases of national importance. New Engl J Med 268:182-192

2. Robert Koch-Institut (RKI) (2013) Surveillance nosokomialer Infektionen sowie die Erfassung von Krankheitserregern mit speziellen Resistenzen und Multiresistenzen. Bundesgesundheitsbl 56(4):580-583

3. Nationales Referenzzentrum (NRZ) für Surveillance von nosokomialen Infektionen (2015) Krankenhaus-Infektions-Surveillance-System (KISS). AMBU-KISS: Surveillance-Protokoll Postoperative Wundinfektionen in Einrichtungen für das ambulante Operieren. https://www.nrz-hygiene. de/fileadmin/nrz/module/ambu/2015_04_ bab_AMBU_KISS_PROTOKOLL_rev_11_fin.pdf. Zugegriffen: 4. Nov. 2019

4. Kommission für Krankenhaushygiene und Infektionsprävention (KRINKO) (2001) Mitteilung der Kommission für Krankenhaushygiene und Infektionsprävention zur Surveillance (Erfassung und Bewertung) von nosokomialen Infektionen (Umsetzung von § 23 IfSG). Bundesgesundheitsbl 44(5):523-536

5. Kommission für Krankenhaushygiene und Infektionsprävention (KRINKO) (2003) Surveillance von postoperativen Wundinfektionen in Einrichtungen für das ambulante Operieren gemäß § 23 Abs. 1 IfSG. Bundesgesundheitsbl 46(9):791-795

6. Storr J, Twyman A, Zingg W et al (2017) Core components for effective infection prevention 
and control programmes: new WHO evidencebased recommendations. Antimicrob Resist Infect Control 6:6

7. Kommission für Krankenhaushygiene und Infektionsprävention (KRINKO) (2010) Die Kategorien in der Richtlinie für Krankenhaushygiene und Infektionsprävention - Aktualisierung der Definitionen. Bundesgesundheitsbl 53(7):754-756

8. Haley RW, Tenney JH, Lindsey JO, Garner JS, Bennett JV (1985) How Frequent Are Outbreaks of Nosocomial Infection in Community Hospitals. Infect Control Hosp Epidemiol 6(6):233-236

9. Wenzel RP, Thompson RL, Landry SM et al (1983) Hospital acquired infections in intensive care unit patients: An overview with emphasis on epidemics. Infect Control 4(5):371-375

10. Kommission für Krankenhaushygiene und Infektionsprävention (KRINKO) (2002) Ausbruchmanagement und strukturiertes Vorgehen bei gehäuftem Auftreten nosokomialer Infektionen. Bundesgesundheitsblatt 45(2):180-186

11. Decker MD (1992) Continuous quality improvement. Infect Control Hosp Epidemiol 13(3):165169

12. Haley RW, Culver DH, White JW et al (1985) The efficacy of infection surveillance and control programs in preventing nosocomial infections in US hospitals. Am J Epidemiol 121(2):182-205

13. Brandt C, Sohr D, Behnke M, Daschner F, Ruden $\mathrm{H}$, Gastmeier P (2006) Reduction of surgical site infection rates associated with active surveillance. Infect Control Hosp Epidemiol 27(12):1347-1351

14. Bärwolff S, Sohr D, Geffers C et al (2006) Reduction of surgical site infections after Caesarean delivery using surveillance. J Hosp Infect 64(2):156-161

15. Geubbels ELPE, Nagelkerke NJD, Mintjes-De Groot AJ, Vandenbroucke-Grauls CMJE, Grobbee DE, De Boer AS (2006) Reduced risk of surgical site infections through surveillance in a network. Int J Qual Health Care 18(2):127-133

16. Gastmeier P, Sohr D, Brandt C, Eckmanns T, Behnke M, Ruden H (2005) Reduction of orthopaedic wound infections in 21 hospitals. Arch Orthop Trauma Surg 125(8):526-530

17. Astagneau P, L'Heriteau F, Daniel F et al (2009) Reducing surgical site infection incidence through a network: results from the French ISO-RAISIN surveillance system. J Hosp Infect 72(2):127-134

18. Marchi M, Pan A, Gagliotti C et al (2014) The Italian national surgical site infection surveillance programme and its positive impact, 2009 to 2011. Euro Surveill 19(20815):21

19. Choi HJ, Adiyani L, Sung J et al (2016) Five-year decreased incidence of surgical site infections following gastrectomy and prosthetic joint replacement surgery through active surveillance by the Korean Nosocomial Infection Surveillance System. J Hosp Infect 93(4):339-346

20. Zuschneid I, Schwab F, Geffers C, Rüden H, Gastmeier P (2003) Reducing central venous catheterassociated primary bloodstream infections in intensive care units is possible: data from the German nosocomial infection surveillance system. Infect Control Hosp Epidemiol 24(7):501-505

21. L'Hériteau F, Olivier M, Maugat S et al (2007) Impact of a five-year surveillance of central venous catheter infections in the REACAT intensive care unit network in France. J Hosp Infect 66:123-129

22. Schwab F, Geffers C, Bärwolff S, Rüden H, Gastmeier P (2007) Reducing neonatal nosocomial bloodstream infections through participation in a national surveillance system. J Hosp Infect 65(4):319-325
23. Schröder C, Schwab F, Behnke M et al (2015) Epidemiology of healthcare associated infections in Germany: Nearly 20 years of surveillance. Int J Med Microbiol 305(7):799-806

24. Zuschneid I, Schwab F, Geffers C, Behnke M, Ruden H, Gastmeier P (2007) Trends in ventilatorassociated pneumonia rates within the German nosocomial infection surveillance system (KISS) Infect Control Hosp Epidemiol 28(3):314-318

25. Gastmeier P, Behnke M, Schwab F, Geffers C (2011) Benchmarking of urinary tract infection rates: experiences from the intensive care unit component of the German national nosocomial infections surveillance system. J Hosp Infect 78(1):41-44

26. Kanerva $M$, Mentula S, Virolainen-Julkunen A et al (2013) Reduction in Clostridium difficile infections in Finland, 2008-2010. J Hosp Infect 83(2):127-131

27. Lytvyn L, Mertz D, Sadeghirad B et al (2016) Prevention of Clostridium difficile Infection: $A$ Systematic Survey of Clinical Practice Guidelines. Infect Control Hosp Epidemiol 37(8):901-908

28. Li Y, Gong Z, Lu Y, Hu G, Cai R, Chen Z (2017) Impact of nosocomial infections surveillance on nosocomial infection rates: A systematic review. Int J Surg 42:164-169

29. Gastmeier P, Schwab F, Sohr D, Behnke M, Geffers C (2009) Reproducibility of the surveillance effect to decrease nosocomal infection rates. Infect Control Hosp Epidemiol 30(10):993-999

30. Schwab F, Gastmeier P, Piening B, Geffers C (2012) The step from a voluntary to a mandatory nationa nosocomial infections surveillance system: the influence on infection rates and surveilance effect. Antimicrob Resist Infect Control 1:24

31. Zingg W, Holmes A, Dettenkofer M et al (2015) Hospital organisation, management, and structure for prevention of health-care-associated infection: a systematic review and expert consensus. Lancet Infect Dis 15(2):212-224

32. Price L, MacDonald J, Melone L et al (2018) Effectiveness of national and subnational infection pre vention and control interventions in high-income and upper-middle-income countries: a systematic review. Lancet Infect Dis 18(5):e159-e171

33. Nationales Referenzzentrum (NRZ) für Surveillance von nosokomialen Infektionen (2018) KISS (Krankenhaus-Infektions-Surveillance-System) Projektbeschreibung. https://www.nrz-hygiene. de/surveillance/kiss/. Zugegriffen: 4. Nov. 2019

34. Robert Koch-Institut (RKI) (2015) Handbuch zum Modul VI der strukturierten curricularen Fortbildung "Krankenhaushygiene". Grundlagen qualitätssichernder Maßnahmen und des Ausbruchsmanagements. Robert-Koch-Institut, Berlin

35. Nationales Referenzzentrum für Surveillance von nosokomialen Infektionen (2017) Definitionen nosokomialer Infektionen für die Surveillance im Krankenhaus-Infektions-Surveillance-System (KISS-Definitionen). https://www.nrz-hygiene. de/fileadmin/nrz/module/KISS Definitionen EBook_Neuauflage_06_2017.pdf.Zugegriffen: 4 . Nov. 2019

36. Garner JS, Jarvis WR, Emori TG, Horan TC, Hughes JM (1988) CDC definitions for nosocomial infections, 1988. Am J Infect Control 16(3):128-140

37. Hansen S, Sohr D, Geffers C et al (2012) Concordance between European and US case definitions of healthcare-associated infections. Antimicrob Resist Infect Control 1:28

38. Kommission für Krankenhaushygiene und Infektionsprävention (KRINKO) (2009) Personelle und organisatorische Voraussetzungen zur Prävention nosokomialer Infektionen. Bundesgesundheitsbl 52(9):951-962

39. Eickhoff TC, Brachmann PS, Bennett JV, Brown $J$ (1969) Surveillance of nosocomial infection in community hospitals. I. Surveillance methods, effectiveness, and initial results. J Inf Dis 120(3):305317

40. Lima NL, Pereira CR, Souza IC et al (1993) Selective surveillance for nosocomial infections in a Brazilian hospital. Infect Control Hosp Epidemiol 14(4):197-202

41. Poulsen KB, Meyer M (1996) Infection registration underestimates the risk of surgical wound infections. J Hosp Infect 1996(33):207-216

42. Schröder C, Behnke M, Gastmeier P, Schwab F, Geffers C (2015) Case vignettes to evaluate the accuracy of identifying healthcare-associated infection sy surveillance persons. J Hosp Infect 90(4):322-326

43. Emori TG, Culver DH, Horan TC et al (1991) National Nosocomial Infection Surveillance System (NNIS): Description of surveillance methodology. Am J Infect Control 19(1):19-35

44. Nationales Referenzzentrum (NRZ) für Surveillance von nosokomialen Infektionen (2016) Protokoll. Surveillance Device-assoziierter nosokomialer Infektionen auf Normalpflegestationen / Nicht-Intensivstationen (STATIONS-KISS). https:// www.nrz-hygiene.de/fileadmin/nrz/module/ station/infektionen/STATIONS-KISS_InfSurv_Protokoll_v20161223.pdf. Zugegriffen: 4. Nov. 2019

45. Edwards J, Peterson K, Andrus M et al (2007) National Healthcare safety Network (NHSN) Report, data summary for 2006, issued June 2007. Am J Infect Control 35(5):290-301

46. European Centre for Disease Prevention and Control (ECDC) Healthcare-associated Infections Surveillance Network (HAl-Net). https://www. ecdc.europa.eu/en/about-us/partnerships-andnetworks/disease-and-laboratory-networks/hainet. Zugegriffen: 4. Nov. 2019

47. Gastmeier P, Sohr D, Just H-M, Nassauer A, Daschner F, Rüden H (2000) How to survey nosocomial infections? Infect Control Hosp Epidemiol 21(6):366-370

48. Puhto T, Syrjälä H (2015) Incidence of healthcareassociated infections in a tertiary care hospital: results from a three-year period of electronic surveillance. J Hosp Infect 90(1):46-51

49. Rhee C, Kadri S, Huang S et al (2016) Objective Sepsis Surveillance Using Electronic Clinical Data. Infect Control Hosp Epidemiol 37(2):163-171

50. Sips M, Bonten M, van Mourik M (2017) Semiautomated surveillance of deep surgical site infections after primary total hip or knee arthroplasty. Infect Control Hosp Epidemiol 38(6):732-735

51. Gastmeier P, Behnke M (2016) Electronic surveillance and using administrative data to identify healthcare associated infections. Curr Opin Infect Dis 29(4):394-399

52. van Mourik M, Perencevich E, Gastmeier P, Bonten M (2018) Designing Surveillance of HealthcareAssociated Infections in the Era of Automation and Reporting Mandates. Clin Infect Dis 66(6):970-976

53. McKibben L, Horan T, Tokars Jl et al (2005) Guidance on public reporting of healthcareassociated infections: recommendations of the Healthcare Infection Control Practices Advisory committee. Infect Control Hosp Epidemiol 26(6):580-587

54. Zuschneid I, Geffers C, Sohr D et al (2007) Validation of surveillance in the intensive care unit component of the German nosocomial infections 
surveillance system. Infect Control Hosp Epidemiol 28(4):496-499

55. Ridgeway S, Wilson J, Charlet A, Kafatos G, Pearson A, Coello $R$ (2005) Infection of the surgical site after arthroplasty of the hip. J Bone Joint Surg Br 87(6):844-850

56. Wilcox M, Shetty N, Fawley W et al (2012) Changing epidemiology of Clostridium difficile infection following the introduction of a national ribotyping-based surveillance scheme in England. Clin Infect Dis 55(8):1056-1063

57. Carlet J, Astagneau P, Brun-Buisson C et al (2009) French national program for prevention of healthcare-associated infections and antimicrobial resistance, 1992-2008: positive trends, but perseverance needed. Infect Control Hosp Epidemiol 30(8):737-745

58. Daneman N, Stukel T, Ma X, Vermeulen M, Guttmann A (2012) Reduction in Clostridium difficile infection rates after mandatory hospital public reporting: findings from a longitudinal cohort study in Canada. PLoS Med 9(7):e1001268

59. Liu H, Herzig C, Dick A et al (2017) Impact of State Reporting Laws on Central Line-Associated Bloodstream Infection Rates in U.S. Adult Intensive Care Units. Health Serv Res 52(3):1079-1098

60. Marsteller J, Hsu Y, Weeks K (2014) Evaluating the impact of mandatory public reporting on participation and performance in a program to reduce central line-associated bloodstream infections: evidence from a national patient safety collaborative. Am J Infect Control 42(10 Suppl):209-215

61. Pakyz A, Edmond M (2013) Influence of state laws mandating reporting of healthcare-associated infections: the case of central line-associated bloodstream infections. Infect Control Hosp Epidemiol 34(8):780-784

62. Richtlinie des Gemeinsamen Bundesausschusses über Maßnahmen zur Qualitätssicherung der Versorgung von Früh- und Reifgeborenen gemäß $\S 136$ Absatz 1 Nummer 2 SGB V in Verbindung mit § 92 Abs. 1 Satz 2 Nr. 13 SGB V (Qualitätssicherungs-Richtlinie Früh- und Reifgeborene/QFR-RL). Fassung vom: 20. Sept. 2005 BAnz. Nr. 205 (S. 15 684) vom 28. Okt. 2005. Letzte Änderung: 22. Nov. 2018 BAnz AT 28. Mai 2019 B1

63. Haustein T, Gastmeier P, Holmes A et al (2011) Use of benchmarking and public reporting for infection control in four high-income countries. Lancet Infect Dis 11(6):471-481

64. Singh S, Davies J, Sabou S, Shrivastava R, Reddy $S$ (2015) Challenges in reporting surgical site infections to the national surgical site infection surveillance and suggestions for improvement. Ann R Coll Surg Engl 97(6):460-465

65. Brunkhorst F, Gastmeier P, Abu Sin M (2018) Aktuelle Aspekte zur Definition und Diagnostik der Sepsis und Antibiotikaresistenz. Bundesgesundheitsbl 61(5):562-571

66. Lee G, Kleinman K, Soumerai S et al (2012) Effect of nonpayment for preventable infections in U.S. hospitals. N Engl J Med 367:1428-1437

67. Meddings J, Reichert H, Rogers M, Saint S, Stephansky J, McMahon L (2012) Effect of nonpayment for hospital-acquired, catheter-associated urinary tract infection: a statewide analysis. Ann Intern Med 157(5):305-312

68. Kadri S, Hohmann S, Zhang F, O'Grady N, Klompas M (2016) 24: Impact of penalties for central line-associated bloodstream infections on blood culture ordering. Crit Care Med 44(12 Suppl):92 


\section{Anhang: Glossar}

\author{
Begriff \\ Querschnitt- Unter- \\ suchung (Endpunkt: \\ Prävalenz) \\ Longitudinal- Unter- \\ suchung (Endpunkt: \\ Inzidenz)
}

Inzidenzdichte

Device-assoziierte Inzidenzdichte

Prospektive Surveillance

Retrospektive Surveillance

Aktive Surveillance

Passive Surveillance

Kontinuierliche Surveillance

Diskontinuierliche Surveillance

Sentinel-Surveillance

Standardisierte Infektionskennzahl (SIK)

Endemische Infektion

Epidemische Infektion
Beschreibung

Eignung für orientierende Untersuchungen zum Vorkommen von einzelnen nosokomialen Infektionen oder zur allgemeinen Sensibilisierung für das Thema Infektionsprävention, da jeder Patient nur einmal erfasst werden muss

- Durch Erfassung der nosokomialen Infektionen von der Krankenhausaufnahme bis zur Entlassung vollständige Verlaufsbeobachtung inklusive Risikofaktorenanalysen

- Nachteil: Zeitaufwand

Berücksichtigung der Expositionsdauer gegenüber dem Risikofaktor Krankenhaus

Berücksichtigung der Expositionsdauer gegenüber "devices" wie Gefäßkatheter, Harnwegskatheter oder Beatmung

- Von einem Starttermin an werden alle während des Beobachtungszeitraumes auftretenden nosokomialen Infektionen erfasst

- Vorteil: alle möglichen Informationsquellen können benutzt und eventuell zusätzliche Untersuchungen veranlasst werden

- Nachteil: hoher zeitlicher Aufwand durch die wiederholte Beurteilung der Patienten während des stationären Aufenthaltes

- Für einen bestimmten Beobachtungszeitraum werden alle aufgetretenen nosokomialen Infektionen zurückschauend erfasst

- Vorteil: geringerer zeitlicher Aufwand, da jede Patientenakte nur einmal durchgesehen werden muss

- Nachteil: Die Patientenakten sind die einzige Informationsquelle (von ihrer Güte hängt die Qualität der Daten ab)

- Die Surveillance wird unter der Regie des Hygienefachpersonals durchgeführt

- Vorteil: wahrscheinlich objektiveres Herangehen durch das Hygienefachpersonal

- Nachteil: Nicht überall ausreichend Hygienefachpersonal vorhanden

- Die Surveillance wird durch den Stationsarzt/niedergelassenen Chirurgen durchgeführt

- Vorteil: Es können auch Befunde und Informationen berücksichtigt werden, die nicht dokumentiert sind

- Nachteil: zusätzliche Arbeitsaufgabe neben vielen anderen, Tendenz zur geringeren Beachtung existiert

- Fortlaufende Erhebung der Infektionsdaten über die Jahre hinweg

- Vorteil: lückenloser Überblick, höhere Präzision der Infektionsraten, Trends analysierbar

- Nachteil: hoher Zeitaufwand

- Zeitlich begrenzte Erfassungsperioden, z. B. zur Evaluation nach Problembehebung oder rotierend

- Vorteil: periodische Sensibilisierung für das Thema

- Nachteil: zufällige Effekte wegen zu kurzer Beobachtungsperioden

Jedes Ereignis wird als Feedback zurückgegeben und soll die Aufmerksamkeit für das Thema steigern (ohne Bezugsdaten)

Ergibt sich als Quotient aus der Anzahl der wirklich beobachteten nosokomialen Infektionen und der nach Risikostruktur der Patientengruppe zu erwarten gewesenen $\mathrm{NI}$
Beispiel

Prävalenz der Blutstrominfektionen in einem Krankenhaus am 1. Juli

Nosokomiale CDI in einem Krankenhaus pro 100 aufgenommene Patienten

Nosokomiale CDI in einem Krankenhaus pro 1000 Patiententage

Beatmungsassoziierte Pneumonien pro 1000 Beatmungstage

Surveillance der Sepsisfälle auf einer neonatologischen Intensivstation

Untersuchung einer Häufung postoperativer Wundinfektionen im Sinne einer Ausbruchsanalyse

Dokumentation postoperativer Wundinfektionen durch Hygienefachkräfte

Dokumentation postoperativer Wundinfektionen durch niedergelassene Chirurgen

Device-assoziierte Infektionen auf allen Intensivstationen in einem Klinikum

Inzidenz der Harnwegsinfektionen bis zur Reduktion einer erhöhten Infektionsrate

Jeder einzelne Fall einer Blutstrominfektion

Die standardisierte Wund-infektionskennzahl hat den Wert 1, wenn die beobachtete Anzahl der Wundinfektionen der erwarteten Anzahl entspricht. Werte der SIK größer als 1 zeigen, dass mehr Infektionen als erwartet auftraten; Werte kleiner als 1 bedeuten, dass weniger Infektionen als erwartet auftraten.

Harnwegskatheter assoziierte nosokomiale Infektionen

Ebola-Epidemie 2014/15 
Sensitivität der Diagnostik von nosokomialen Infektionen

Spezifität der Diagnostik von nosokomialen Infektionen

Stratifizierung
- Korrekte Erkennung, dass es sich um eine nosokomiale Infektion handelt (richtig positiv)

- Korrekte Erkennung, dass es sich nicht um eine nosokomiale Infektion handelt (falsch negativ)

- Es werden separate Infektionsraten für bestimmte Patientengruppen berechnet
$80 \%$ bedeutet, dass 2 von 10 nosokomialen Infektionen übersehen wurden

$100 \%$ bedeutet, dass in keinem Fall ein Patient als nosokomial infiziert eingestuft wurde, der nicht auch wirklich eine nosokomiale Infektion hatte

ZVK-assoziierte Blutstrominfektionsraten für Frühgeborene der Geburtsgewichtsklasse 500 bis $999 \mathrm{~g}$ 